\title{
Effect of Na-Substitution on Magnetoresistance and flux pinning energy of Bi-2212 Ceramics prepared via Hot-Forging Process \\ M.Gürsul*a, B. Özçelik ${ }^{\mathrm{a}}$, A. Sotelo ${ }^{\mathrm{b}}$, M. A. Madre ${ }^{\mathrm{b}}$
}
a Department of Physics, Faculty of Sciences and Letters, Çukurova University. 01330 Adana, Turkey
b ICMA (CSIC-Universidad de Zaragoza). María de Luna, 3. 50018 Zaragoza, Spain.

\begin{abstract}
s
In this study, polymerization method with polyethyleneimine, followed by hot-forging process was used to synthesize bulk textured $\mathrm{Bi}_{2} \mathrm{Sr}_{2} \mathrm{Ca}_{1-x} \mathrm{Na}_{x} \mathrm{Cu}_{2} \mathrm{O}_{y}$ (x=0.0, 0.05, 0.075 0.1, and 0.15) ceramics. Magnetoresistance performance of samples was studied by change of flux pinning mechanism. The effect of Na-substitution on the magnetoresistance, flux pinning energy, irreversibility field, upper critical magnetic field and coherence length was evaluated in the framework of Thermally Activated Flux Flow (TAFF) model. A resistivity transition broadening under various magnetic fields (0-5 T) was analyzed. By using the resistivity data, the upper critical field and the coherence length at $T=0 \mathrm{~K}$ were deduced. For $H \perp c, H_{C 2}(0)$ and $\xi(0)$ values were calculated as $31,31.3,36.7,38.3,35.1 \mathrm{~T}$ and $33.1,32.4,30.0,29.3,30.6$ $\AA$, for $0.0,0.05,0.075,0.10$, and 0.15 Na-doped samples, respectively. For $\mathrm{H} / / \mathrm{c}, H_{C_{2}}(0)$ and $\xi(0)$ values were 95 and $112.3 \mathrm{~T}$ and 18.6 and $17.1 \AA$, for the samples of $\mathrm{Na} 0$ and $\mathrm{Na} 2$, respectively. In particular, the flux pinning or activation energies of $\mathrm{Bi}_{2} \mathrm{Sr}_{2} \mathrm{Ca}_{1-\mathrm{x}} \mathrm{Na}_{\mathrm{x}} \mathrm{Cu}_{2} \mathrm{O}_{8+\mathrm{y}}$ where $x=0.10$ were determined to be $0.19 \mathrm{eV}$ for $0 \mathrm{~T}$ and $0.06 \mathrm{eV}$ for $5 \mathrm{~T}$.
\end{abstract}

Keywords: High $\mathrm{T}_{\mathrm{c}}$ superconductors; Magnetoresistivity; Flux pinning energy; Upper critical magnetic field; Coherence length; Hot-forging technique

*Corresponding Author: Tel./fax:+90.322.3386060/2496/+90.322.3386070

e-mail: ozcelik@cu.edu.tr 


\section{Introduction}

Bi-based superconductors $\mathrm{Bi}_{2} \mathrm{Sr}_{2} \mathrm{Ca}_{n-1} \mathrm{Cu}_{n} \mathrm{O}_{x}$ where $n=1$, 2, and 3, (BSCCO) discovered by Maeda et al. [1] are characterized by a relatively high superconducting transition temperature $T_{\mathrm{C}} \sim 20,90$ and $110 \mathrm{~K}$ for the phases with $n=1,2$, and 3, respectively. Moreover, the highest upper critical magnetic field, $H_{c 2}$, can reach $150 \mathrm{~T}$. In the last decades, the interest on these materials was increased day by day owing to the fact that BSCCO superconductors are the most promising materials for potential industrial applications such as superconducting wires, tapes, resonators, single charge devices, antennas, and switches. On the other hand, there are still many problems to deal with for their technological applications.

In order to improve the physical and magnetic properties of BSCCO system many substitutions have been made and various preparation techniques have been examined [2-22]. Flux pinning is a well-known phenomenon implying that magnetic flux lines are trapped, or pinned, in spite of the Lorentz force acting on them inside a current-carrying type-II superconductor. The motion of vortices in type-II superconductors is hindered by pinning forces associated with various structural defects. The efficient pinning centers are formed by extended growth defects, such as edge dislocations, twin planes, and grain boundaries, as well as by artificial, radiation-induced columnar defects [23,24]. The nature of pinning sites in superconducting samples mainly depends on the grain boundaries or impurities resulting from the substitution into the matrix [23-26]. In order to prevent flux creep creating a pseudoresistance, flux pinning is a desirable phenomenon in HTc superconductors. It should be emphasized at this point that vortex pinning is a very important tool for maintaining the nondissipative transport currents. The pinning of vortices can be achieved either by natural pinning centers like imperfections, defects and grain boundaries or by artificial pinning centers represented in the form of chemical doping, neutron irradiation and heavy ion irradiation [27-29].

For the technological applications of HTc superconductors, the most important parameter is the critical current density. The maximum current density driving through a superconductor without dissipation is determined by the flux pinning mechanism. Dissipating energy in superconductors means flux motion [30]. For understanding the resistivity broadening under an applied magnetic field, there are several models; as flux flow [31], thermally activated flux creep [32], flux line melting and flux cutting [33], etc. The Lorentz force exerted on the flux bundle is smaller than the pinning force, but the flux line may be thermally activated over the 
pinning energy barrier. Most of the scientists have reported that a thermally activated flux creep model can describe the data in the resistive region near $T_{\mathrm{C}}(\rho=0)$ [34-37].

On the other hand, for obtaining bulk high-Tc monoliths with well-oriented grains, one successfull way is using the hot-forging method [20,38]. Through this technique, it is easy to obtain a good grain orientation perpendicular to the press axis. The critical current, $J \mathrm{c}$, values can be enhanced when measured along the preferential conducting plane ( $a b)$. Recently, many experiments related to this technique have already been performed on various kind of samples using different heat treatments and pressures. In these studies, the results depended on the applied temperature used during the pressing process. If the applied temperature is too low to form a minimal liquid phase, the technique has not the pre-conditions to produce high densification and grain orientation. On the other hand, if the temperature is too high, a melting process will be occurred, resulting to glassy materials via quenching [38].

In previous studies, it has been reported that when the samples are produced by partial or total melting, $\mathrm{Na}$ substitution for $\mathrm{Ca}$ in $\mathrm{Bi}-2212$ system provides high $T c$ values $[18,39,40]$. In addition, the polymer solution technique involving polyethyleneimine (PEI) is also useful to enhance the critical temperature, $T c$, in the Bi-2212 phase [41]. Moreover, Bi-2212 bulk sintered, textured and sintered-forged ceramics with different $\mathrm{Na}$ content, produced via PEI, Laser Floating Zone (LFZ) and sintered-forging techniques, respectively, were worked out $[13,18]$. In the framework of this study, we intend to investigate the magnetoresistive properties of the $\mathrm{Bi}_{2} \mathrm{Sr}_{2} \mathrm{Ca}_{1-\mathrm{x}} \mathrm{Na}_{\mathrm{x}} \mathrm{Cu}_{2} \mathrm{O} x$ with $\mathrm{x}=0.0,0.05,0.075,0.10$, and 0.15 textured materials produced via the hot-forging method. We will try to explain the experimental findings by using the thermally activated flux creep model on the magnetoresistance curves measured at different magnetic fields between 0-5 $\mathrm{T}$.

\section{Experimental}

Polycrystalline precursors of $\mathrm{Bi}_{2} \mathrm{Sr}_{2} \mathrm{Ca}_{1-\mathrm{x}} \mathrm{Na}_{\mathrm{x}} \mathrm{Cu}_{2} \mathrm{O}_{\mathrm{x}}$ where $\mathrm{x}=0.0,0.05,0.075,0.10$, and 0.15 were produced by using a polymer solution method [10,20]. The details of sample preparation were given in elsewhere [20]. Powder XRD patterns of textured ceramic samples were taken by using a Rigaku D/max-B X-ray powder diffractometer ( $\mathrm{CuK} \alpha$ radiation) with $2 \theta$ ranging between 3 and 80 degrees. Magnetoresistance was determined through the conventional fourpoint probe technique under different magnetic fields $(0,1,3$, and $5 \mathrm{~T})$. Zero field cooling (ZFC) procedure was used during all measurements. The samples with $\mathrm{x}=0.0,0.05,0.075$, 0.1 , and 0.15 will be hereafter named as $\mathrm{Na} 0, \mathrm{Na} 1, \mathrm{Na} 2, \mathrm{Na} 3$, and $\mathrm{Na} 4$, respectively. 


\section{Results and discussion}

Powder XRD patterns of samples between $3^{0}-80^{0}$ are given in Fig. 1. The most intense peaks are related to the $(00 l)$ planes of Bi-2212 phase, pointing out that the grains have their $c$-axis oriented perpendicular to the sample holder surface. This effect has its origin in the large dimensions of grains along the $a$ - and $b$-directions which promotes their preferential orientation in the samples preparation, as previously observed [13,18]. A pseudo-tetragonal structure with $a-b \approx 5.40 \dot{\mathrm{A}}$ and $c=30.88 \dot{\mathrm{A}}$ has been determined. It may be concluded that $\mathrm{Na}$ substitution is an useful tool to increase the grain sizes, resulting in lower number of grain boundaries. Moreover, the substitution of $\mathrm{Na}$ for $\mathrm{Ca}$ in $\mathrm{Bi}-2212$ phase causes a decrease in the melting point, inducing the formation of a liquid phase, speeding up the diffusion rate, and producing larger grains. As it is well known, obtaining single Bi-2212 phase is very difficult, and it is always accompanied by the Bi-2201 phase (indicated by * in the graph). Additionally, the $\mathrm{SrCuO}_{2}$ peaks, indicated by + in the plot with space group Cmcm, have also been identified, in agreement with previous works [44].

As it is well-known, in order to better understand the doping mechanism influence on the transition temperature, the empirical formula for the calculation of the charge carrier concentration, $p$, in the $\mathrm{CuO}_{2}$ planes has been suggested by Presland et al. [45]. The calculated hole concentration values for the samples are tabulated in Table-1, where it can be seen that all values are close to each other. These results point out that the amount of $\mathrm{Na}$ content is far from the over-doping limit, in agreement with the literature [46].

Thermoelectric power, $S$, of high- $T_{c}$ superconductors depends on the charge carrier transport mechanism . Therefore, the theoretical calculation of $S$ in the Bi-based system, with respect to carrier concentration and temperature was performed [47-49]. According to Cooper et al. [50], the thermoelectric power of $H T c$ materials is given by:

$$
\left.\left.S=k_{B} / e[\ln (1-p) / 2 p)-\ln 2\right)\right]
$$

where $p, k_{B}$ and $e$ are the hole concentration, Boltzmann constant and electron charge, respectively. Using the $p$-values given in Table-1, the $S$ values for the samples have been calculated and displayed in Table-1. As it can be seen, all the values are positive and point out to a dominating hole conduction mechanism.

The magnetic field dependence of resistivity with respect to the temperature is presented, in the range of $160 \mathrm{~K}$ down to $20 \mathrm{~K}$, in Figure-2. The critical onset $\left(T_{c}^{\text {onset }}\right)$ and offset $\left(T_{c}^{\text {offset }}\right)$ temperatures are evaluated from the magnetoresistance graphs given in Fig. 2 and tabulated in Table 2. As it is well-known, while $T_{c}^{\text {onset }}$ temperature concerns the transition of isolated grains to the superconducting state, $T_{c}^{\text {offset }}$ temperature is related to the volume 
fraction of Bi-2212 phase and/or the characteristics of the intergranular components. As can be seen from Fig.2, the onset transition temperature is not obviously affected by the applied magnetic field due to the absence of flux traps and the increment of the superconducting volume fraction, together with the number of charge carriers. It can be argued that the flux pinning is ineffective in the intragranular region. At zero magnetic field $T_{c}^{\text {onset }}$ temperature values increase from $100.2 \mathrm{~K}$ to $101.4 \mathrm{~K}$. This small variation of the onset temperature values arises from the increment in the number of charge carriers in the $\mathrm{CuO}_{2}$ planes. On the other hand, the resistivity of the samples is strongly dependent upon the applied magnetic field in the vicinity of the zero resistivity transition temperature, $T_{c}^{o f f s e t}$, as a consequence of the dissipative regime of vortex motion. This is a clear evidence of more superconducting charge carriers at lower temperatures. Hence it may be concluded that the vortices pinning is highly effective and the applied magnetic field affects more the intergrain transitions than the intragrain ones, pointing out the low granularity structure in the studied samples. In addition, because of the onset transition temperature $\left(T_{c}^{\text {onset }}\right)$ is observed above $90 \mathrm{~K}$, it may be argued that the $\mathrm{Bi}-2212$ phase is the dominant one in the ceramic matrix.

In order to observe the anisotropic problem, we have applied the magnetic field perpendicular to the $a b$-plane (parallel to $c$-plane). The temperature dependence of resistivity of $\mathrm{Na} 2$ sample for zero field and $5 \mathrm{~T}$ applied magnetic field parallel to the $c$-plane is shown in Fig.3. As can be seen from Table-2, while the onset and offset temperatures are slightly changing for the zero field, for the $5 \mathrm{~T}$ applied magnetic field, the offset temperature is drastically increasing (almost $20 \mathrm{~K}$ ) when measuring parallel to $c$-axis.

In order to better understand the effect of $\mathrm{Na}$ substitution on the activation energy, irreversibility field and coherence length of the samples, we have also studied the TAFF model. According to this model, the resistivity at the tail part broadens and obeys the Arrhenius relation given in [51]

$$
\rho(H, T)=\rho_{0} \exp \left(-U(H) / k_{B} T\right)
$$

where $U$ is the flux pinning or activation energy that depends on temperature and magnetic field. The broadening tail part is extremely sensitive to the magnetic field even for small values and proportional to the magnitude and strength of pinning force. The flux pinning or activation energy, $U$, value can be directly determined from the slope of the $\ln \left(\rho / \rho_{0}\right)$ versus $1 / T$ plot given in Fig. 4. From these figures, a very obvious shifting phenomenon towards lower temperatures is generally observed by increasing the applied magnetic field. This is a well-known behavior attributed to the flux flow resistivity i.e. lowering the temperature 
increases the pinning strength, hence the pinning force. In addition, there is nearly an exponential dependence of $\rho$ on $1 / T$ for $T<<T_{c}^{\text {onset }}$, pointing out to the energy dissipation in the TAFF region due to the thermal activation of fluxes across the pinning barriers. Therefore, it is possible to deduce the $U$ values from the slopes of straight line portions of the curves at low temperatures. In Fig.5, the flux pinning energy, $U$, versus applied magnetic field is exhibited for all samples. As can be seen, the $U$ values rapidly decreased by increasing the applied magnetic field. In addition, the flux pinning energies of samples significantly increase with the enhancement of $\mathrm{Na}$ content. It may be concluded that the enhancement of the energy barriers originates from the increment of Na-content causing an increase of the number of pinning centers. These pinning centers behave as effective pinning centers, hence the penetration of the applied magnetic field into the sample is not allowed. In addition, the high critical current density, $J c$, values are found, pointing out the evidence of the strong pinning mechanism originated from Na-substitution.

In this case, an increment of the intergranular coupling and a decrease of the number of weak links are revealed and the vortices cannot easily creep because of the relatively large activation energy values, with a reduction of the weak links. In addition, it will be worthwhile to emphasize that below $5 \mathrm{~T}$, the applied magnetic field may penetrate the intergranular region, ascribing to the existence of different superconducting levels like the superconducting grains, the weak-links, and the superconducting clusters within the samples in these regions. Thus, it can be concluded that the interconnected grains fraction by strong-links in sample $\mathrm{Na} 3$ is very large in comparison with those of the other ones.

The magnetic field dependence of flux pinning energy follows a power-law given as;

$$
U(H) \approx H^{-\beta}
$$

where $\beta$ is a magnetic field constant orientated with respect to basal plane [52]. By using the data presented in Fig.5, we have obtained $\beta$ values of $0.62,0.63,0.64,0.61$, and 0.57 (see Table-1) for samples $\mathrm{Na} 0-\mathrm{Na} 4$, respectively.

The values of $\beta$ for Na-substituted samples are similar to the values obtained for Mnsubstituted $\mathrm{BiPb}-2223$ samples $(\beta=0.45-0.61)$ [53]. As a result, there are no predictable values of $\beta$ since they depend on many factors affecting the pinning energy and the characteristics of crystal structure, like the real atomic positions, quality of weak links and oxygen content [52].

In addition, the magnetic field dependence on the transition width $\Delta T\left(=T_{c}^{90 \%}-T_{c}{ }^{10 \%}\right)$ follows the power-law relation as;

$$
\Delta T \approx H^{n} \approx H^{\beta / q}
$$


The $\Delta T$ values for different Na-content versus the magnetic field are given in Fig.6. As can be seen $\Delta T$ increases with increasing $\mathrm{H}$ values. This increment in $\Delta T$ by increasing $H$ can be explained taking into account that during cooling time, a number of grains and clusters in random positions freeze, leading to some more unwanted resistive regions in all samples. Accordingly, these regions cause the weakening of vortex pinning strength due to the reduction of the superconducting current path [52]. On the other hand, the decrease in $\Delta T$ by increasing the Na-content might be due to the decrease in microscopic inhomogeneous superconducting phases and/or an increment of intergrain connectivity. From this experimental data and by drawing log-log plot of $\Delta T$ versus magnetic field, $n$ and $q$ values are calculated and tabulated in Table 1. By looking at Table-1, it is easy to see that Na2 sample has the highest the $\beta, q$ and $n$ values.

Finally, according to thermally activated flux creep (TAFC) model and AmbegaokarHalperin $(\mathrm{AH})$ theory, $U(H, T)$ can be given as [54,55];

$$
U(H, T) \approx \Delta T H^{-\eta}
$$

where $\eta=\beta+n$. The $\eta$ values are also tabulated in Table- 2 .

The irreversibility line (IL) is believed to display the change from the flux pinning dominated regime to that dominated by the TAFF, where resistance occurs. The irreversibility magnetic field, $H_{i r r}$, values versus offset temperatures finding by considering $10 \%$ of normal state resistivity has been deduced from the resistivity measurements and plotted in Figure 7, for all the samples. When the irreversibility limits of the samples for different applied fields are plotted in an $H-T$ diagram, an IL is generated separating the $H-T$ plane into two regions: a low temperature region, in which the flux dynamics is dissipative and thus irreversible, and a high temperature one, where the flux motion is free and magnetically reversible. In Figure 7, a gradual change in $H_{i r r}$ in the vicinity of $T_{c}$ by decreasing temperature, followed by a rapid increase with strong curvature at lower temperatures, is observed. Additionally, comparing the position of the irreversibility field lines, IL shifts towards the high temperature values with increasing Na-content, indicating that the strength vortex fluctuations with increasing Nacontent.

Taking the midpoint values of $T_{c}^{\text {onset }}$ and $T_{c}^{\text {offset }}$ temperatures of Fig. 2, the upper critical field, $H_{c 2}(T)$, values are plotted in Fig. 8. For determining the upper critical field in the vicinity of zero temperature, the formula given by Werthamer-Helfand-Hohenberg (WHH) [56]

$$
H_{c 2}(0)=0.693 T_{c}\left(d H_{c 2}(T) / d T\right)_{T c}
$$


was employed, where the $\left(d H_{c 2}(T) / d T\right)$ are obtained by using $T_{C}$ values determined from the midpoint of the linear fit of the highest slope of the resistivity curves at zero field given in Figure 2.

In the superconductors, the coherence length, $\boldsymbol{\xi}$, is another important parameter. $\boldsymbol{\xi}$ was calculated by using the Ginzburg-Landau relation given as [57],

$$
\mu_{0} H_{c 2}(0)=\Phi_{0} / 2 \pi \xi^{2}(0)
$$

where $\Phi_{0}$ is the quanta of flux $\left(2.07 \times 10^{-15} \mathrm{~T} . \mathrm{m}^{2}\right)$ and $H_{c 2}(0)$ is the upper critical field at zero temperature. The calculated $H_{c 2}$ and $\xi$ values are given in Table-1. As can be seen, for sample $\mathrm{Na} 2$, the calculated $H_{c 2}$ and $\xi$ values at zero $\mathrm{K}$ for $H^{\perp_{c}}$ and $\mathrm{H} / / \mathrm{c}$ are 36.7 and $112.3 \mathrm{Tesla}$, and 30 and $17.1 \AA$, respectively.

\section{CONCLUSIONS}

Polycrystalline $\mathrm{Bi}_{2} \mathrm{Sr}_{2} \mathrm{Ca}_{1-x} \mathrm{Na}_{x} \mathrm{Cu}_{2} \mathrm{O}_{8+y}$ superconductors ( $x=0.0,0.05,0.075,0.10$, and 0.15 ) have been produced by the polymerization route followed by hot-forging technique. From XRD patterns, it may be seen that the most intense peaks belong to the (00l) planes of Bi2212 phase. A pseudo-tetragonal structure with $a-b \approx 5.40 \dot{\mathrm{A}}$ and $c=30.88 \dot{\mathrm{A}}$ have been obtained for the crystal symmetries. The calculated thermoelectric power $(S)$ values point out to dominating hole conduction mechanism in these samples. The magnetoresistance results have been used in order to study the variations in the flux pinning mechanism. The activation energies, irreversibility fields, upper critical fields and coherence lengths in the vicinity of $0 \mathrm{~K}$ have been deduced from the $\rho-T$ data. The broadening behavior of resistivity under different magnetic fields has been studied by using the TAFF model. The enhancement of the thermoelectric power, the derived flux pinning energy, and upper critical field, $H_{c 2}$, by increasing $x$ up to 0.10 has also been reported. A reverse trend has been found with increasing of Na-content. This may be accepted as an evidence of the enhancement of the energy barriers originated from the increment of Na-content causing an increasing of the pinning centers. These pinning centers act as effective pinning centers, therefore, the penetration of the applied magnetic field is not allowed into the sample. In addition, the $J_{C}$ values are found to point out the evidence of the strong pinning mechanism originated from Na-substitution. In this case, an increment of the intergranular coupling and a decrease of the number of weak links are revealed and the vortices cannot easily creep because of the relatively large activation energy values, with a reduction of the weak links. 


\section{Acknowledgements}

This work is supported by the Research Fund of Çukurova University, Adana, Turkey, under grant contracts no. FBA-2014-3500. M.A. Madre, and A. Sotelo wish to thank the Gobierno de Aragón-FEDER (Research Group T 54-17 R), and the Spanish MINECO-FEDER (MAT2017-82183C3-1-R) for financial support. Authors would like to acknowledge the use of Servicio General de Apoyo a la Investigación-SAI, Universidad de Zaragoza.

\section{References:}

[1] H.Maeda, Y. Tanaka, M. Fukutomi, T. Asano, Jpn. J. Appl. Phys. 27, L 209 (1988)

[2] B.Özkurt, B.Özçelik, J. Low Temp.Phys. 156, 22 (2009)

[3] D.Yazici, M.Erdem, B.Özçelik, J. Supercond. Nov.Magn. 25, 1811 (2012)

[4] G. F. de la Fuente, A. Sotelo, Y. Huang, M. T. Ruiz, A. Badia, L. A. Angurel, F. Lera, R. Navarro, C. Rillo, R. Ibañez, D. Beltran, F. Sapiña, A. Beltran, Physica C, 185, 509 (1991)

[5] B. Özçelik, B. Özkurt, M.E. Yakıncı, A. Sotelo, M. A. Madre, J. Supercond. Nov. Magn., 26, 873 (2013)

[6] H. Gündogmus, B. Özçelik, B. Özkurt, A. Sotelo, M.A. Madre, J. Supercond. Nov. Magn, 26, 111 (2013)

[7] A. Ozaslan, B. Özçelik, B. Özkurt, A. Sotelo, M.A. Madre, J. Supercond. Nov. Magn, 27, 53 (2014)

[8] C. Kaya, B. Özçelik, B. Özkurt, A. Sotelo, M.A. Madre, J Mater Sci:Mater Electron, 24, $1580(2013)$

[9] B. Ozcelik, M. Gursul, A. Sotelo, M. A. Madre, J Mater Sci: Mater Electron. 25(10): 4476 (2014)

[10] A. Sotelo, Sh. Rasekh, M.A. Madre, J.C. Diez: J. Supercond. Nov. Magn. 24, 19 (2011)

[11] B. Ozcelik, M. Gursul, A. Sotelo, M.A. Madre, J Mater Sci: Mater.Electron 26, 441 (2015)

[12] B. Ozcelik, M. Gursul, A. Sotelo, M.A. Madre, J Mater Sci:Mater Electron., 26, 2830 (2015)

[13] M. Gursul, A.Ekicibil, B. Ozcelik, A. Sotelo, M. A. Madre, J Supercond Nov Magn 28:1913 (2015)

[14] B. Ozcelik, A.Tangüner, M. Gursul, A. Sotelo, M. A. Madre, J Supercond Nov Magn. $28: 2641(2015)$

[15] B. Özkurt, B. Özçelik, M. A. Madre, A. Sotelo, J. C. Diez, J Mater Sci: Mater Electron 26:5761 (2015) 
[16] A. Sotelo, B. Ozcelik, H. Amaveda, A. Bruned, M. A. Madre, Ceramics International, 41, 14276, (2015).

[17] B. Özçelik, O. Nane, A. Sotelo, M.A. Madre, Ceramics International 42, 3418 (2016)

[18] O. Nane, B. Özçelik, H. Amaveda, A. Sotelo, M.A. Madre, Ceramics International, 42, 8473 (2016)

[19] O. Nane, B. Özçelik, J Mater Sci: Mater Electron., 27/7, 7633, (2016)

[20] O. Nane, B. Özçelik, A. Sotelo, M.A. Madre, J. Eur. Ceram Soc., 37, 1007 (2017)

[21] B. Özçelik, O. Nane, A. Sotelo, H. Amaveda, M.A. Madre J Mater Sci: Mater Electron., 28, 6278 (2017)

[22] E. Gün, B. Özkurt, B. Özçelik, M. A. Madre, A. Sotelo, J. C. Diez, J Mater Sci: Mater Electron. 28, 13120 (2017)

[23] J. M. Huijbregtse, B. Dam, R. C. F. van der Geest, F. C. Klaassen, R. Elberse, J. H.Rector, and R. Griessen, Phys. Rev. B 62, 1338 (2000)

[24] J. Albrecht, Phys. Rev. B 68, 054508 (2003)

[25] M. Hawley, I. D. Raistrick, J. G. Beery, and R. J. Houlton, Science 251, 1587 (1991)

[26] C. Gerber, D. Anselmetti, J. G. Bednorz, J. Mannhart, and D. G. Schlom, Nature (London) 350, 279 (1991)

[27] J. Trastoy, V. Rouco, C. Ulysse, R. Bernard, G. Faini, J. Lesueur, J. Briatico, J.E. Villegas,

Physica C 506, 195, (2014)

[28] M. Shahbazi, X.L. Wang, S.R. Ghorbani, M. Ionescu, O.V. Shcherbakova, F.S. Wells, A.V. Pan, S.X. Dou, K.Y. Choi, Supercond. Sci. Technol., 26: 095014 (2013)

[29] M. Eisterer, M. Zehetmayer, H.W. Weber, J. Jiang, J.D. Weiss, A. Yamamoto, E.E. Hellstrom, Supercond. Sci. Technol., 22: 095011, (2009)

[30] P.W. Anderson, Phys.Rev.Lett. 9,309 (1962)

[31] M.Tinkham, Phys. Rev. Lett. 61, 1658 (1988)

[32] T.T.Palstra, B.Batlogg, R.B. Van Dover, L.F. Scheemeyer, J. V.Waszczak, Appl. Phys. Lett. 54, 763 (1989)

[33] M.R.Mohammadizadeh, M.Akvahan, Physica C 390, 134 (2003)

[34] T.T. Palstra, B. Batlogg, L.F. Schneemeyer, J.V. Waszczak, Phys. Rev. Lett. 61 $1662(1988)$

[35] A.P. Malozemoff, T.K. Worthington, E. Zeldov, N.C. Yeh, M.W. McElfresh, in Strong Correlation and Superconductivity, ed. by H. Fukuyama, S. Maekawa, A.P. Malozemoff. Springer Series in Sol.State Sci., vol. 89, Springer, Berlin, (1989) 
[36] R.Griessen, Phys. Rev. Lett. 64, 1674 (1990)

[37] R.C. Ma, W.H. Song, X.B. Zhu, L. Zhang, S.M. Liu, J. Fang, J.J. Du, Y.P. Sun, C.S. Li, Z.M. Yu, Y. Feng, P.X. Zhang., Physica C 405, 34 (2004)

[38] V. Garnier, R. Caillard, A. Sotelo, G. Desgardin, Physica C 319, 197 (1999)

[39] S.-X. Dou, W.-M. Wu, H.-K. Liu, C. C. Sorrell, Physica C 185-189, 811 (1991)

[40] Y. Yu, X. Jin, D. X. Cai, X. X. Yao, C. Hu, K. Y. Ding, D. Feng, Phys. Stat. Sol. A 146, K33-K36 (1994)

[41] A. Sotelo, J. I .Peña, L. A. Angurel, C. Diez, M. T. Ruiz, G. F. de la Fuente, R. Navarro, J. Mater. Sci. 32, 5679 (1997)

[42] A. Sotelo, H. Szillat, P. Majewski, F. Aldinger, Supercond. Sci. Technol. 10, 717 (1997)

[43] A. Sotelo, M. Mora, M. A. Madre, J. C. Diez, L. A. Angurel, G. F. de la Fuente, J. Eur. Ceram. Soc. 25, 2947 (2005)

[44] N. N. Eremin, L. I. Leonyuk, V. S. Urusov, J. Solid State Chem. 158, 162 (2001)

[45] M.R.Presland, J.L. Tallon, R.G. Buckley, R.S. Liv, N.E. Floer, PhysicaC, 176, 95 (1991)

[46] S. Satyavathi, K. Nanda Kishore, V. Hari Babu, O. Pena, Sup. Sci.Tech., 9, 93 (1996)

[47] M.C. Sekhar and S.V. Suryanarayana, Physica C, 415, 209 (2004)

[48] G. Beni, Phys. Rev. B10, 2187 (1974)

[49] P.M.Chaikin, G.Beni, Phys. Rev.B 46, 647 (1976)

[50] J.R. Cooper, B. Alavi, L.W. Zhow, W.P. Boyermann, G. Gruner, Phys. Rev. B 35, $8794(1987)$

[51] J.J. Kim, H. Lee, J. Chung, H.J. Shin, H.J. Lee, J.K. Ku, Phys. Rev. B 43, 2962 (1991)

[52] M.M. Barakat, K. Habanjar, J. of Advanced Ceramics, 6(2), 100 (2017)

[53] M. Dogruer, Y. Zalaoglu, A. Varilci, C. Terzioglu, G. Yildirim, O. Ozturk, J. Supercond Nov Magn, 25, 961 (2012)

[54] H. Khosroabadi, V. Daadmehr, M. Akhavan, Physica C, 384, 169 (2003)

[55] N.H. Mohammed, A.I. Abou-Aly, R. Awad, M. Rekaby, Sup. Sci.Tech, 19, 1104 (2006)

[56] N.R. Werthamer, E. Helfand, P.C. Hohenberg, Phys. Rev. 147, 295 (1966)

[57] H.C. Yang, L.M. Wang Phys Rev B 59, No:13 (1999) 
Table 1. Physical properties of all samples

\begin{tabular}{|l|c|l|l|c|l|l|l|l|}
\hline Sample & $\mathrm{P}$ & $\mathrm{S}(\mu \mathrm{V} / \mathrm{K})$ & $H_{c 2}(\mathrm{~T})$ & $\xi(\AA)$ & $\beta$ & $n$ & $\eta$ & $q$ \\
\hline Na0 & 0.1935 & 3.51 & $31 \mathrm{H} \perp c$ & $33.1 \mathrm{H} \perp c$ & 0.62 & 0.21 & 0.83 & 3.01 \\
& & & $95 \mathrm{H} / c$ & $18.6 \mathrm{H} \| c$ & & & & \\
\hline Na1 & 0.1933 & 3.65 & $31.3 \mathrm{H} \perp c$ & $32.4 \mathrm{H} \perp c$ & 0.63 & 0.21 & 0.84 & 2.79 \\
\hline Na2 & 0.1931 & 3.76 & $36.7 \mathrm{H} \perp c$ & $30.0 \mathrm{H} \perp c$ & 0.64 & 0.22 & 0.86 & 2.94 \\
& & & $112.3 \mathrm{H} / / c$ & $17.1 \mathrm{H} \| c$ & & & & \\
\hline Na3 & 0.1928 & 3.93 & $38.3 \mathrm{H} \perp c$ & $29.3 \mathrm{H} \perp c$ & 0.61 & 0.21 & 0.82 & 2.91 \\
\hline Na4 & 0.1933 & 3.65 & $35.1 \mathrm{H} \perp c$ & $30.6 \mathrm{H} \perp c$ & 0.57 & 0.20 & 0.77 & 2.79 \\
\hline
\end{tabular}

Table 2. $\mathrm{T}_{\mathrm{c}}{ }^{\text {onset }}$ and $\mathrm{T}_{\mathrm{c}}{ }^{\text {offset }}$ values of samples at various applied magnetic fields deduced from the $R-T$ measurement data

\begin{tabular}{|c|c|c|c|c|c|}
\hline Sample & Temp. & 0 Tesla & 1 Tesla & 3 Tesla & 5 Tesla \\
\hline $\mathrm{NaO} \quad(\mathrm{H} \perp \boldsymbol{c})$ & $\begin{array}{l}T_{c}^{\text {onset }} \\
T_{\text {offset }}^{\mathrm{c}}\end{array}$ & $\begin{array}{l}100.2 \mathrm{~K} \\
84.2 \mathrm{~K}\end{array}$ & $\begin{array}{l}100.1 \mathrm{~K} \\
58.1 \mathrm{~K}\end{array}$ & $\begin{array}{l}100.0 \mathrm{~K} \\
46.7 \mathrm{~K}\end{array}$ & $\begin{array}{l}99.6 \mathrm{~K} \\
41.3 \mathrm{~K}\end{array}$ \\
\hline Na1 $(\mathrm{H} \perp \boldsymbol{c})$ & $\begin{array}{l}\mathrm{T}_{\mathrm{c}}^{\text {onset }} \\
\mathrm{T}^{\mathrm{c}}{ }_{\text {offset }}\end{array}$ & $\begin{array}{l}100.3 \mathrm{~K} \\
84.8 \mathrm{~K}\end{array}$ & $\begin{array}{l}100.2 \mathrm{~K} \\
58.8 \mathrm{~K}\end{array}$ & $\begin{array}{l}100.1 \mathrm{~K} \\
48.1 \mathrm{~K}\end{array}$ & $\begin{array}{l}99.7 \mathrm{~K} \\
41.9 \mathrm{~K}\end{array}$ \\
\hline Na2 $(\mathrm{H} \perp c)$ & $\begin{array}{l}\mathrm{T}_{\mathrm{c}}^{\text {onset }} \\
\mathrm{T}_{\text {offset }}^{\mathrm{c}}\end{array}$ & $\begin{array}{l}101.2 \mathrm{~K} \\
85.7 \mathrm{~K}\end{array}$ & $\begin{array}{l}101.0 \mathrm{~K} \\
62.7 \mathrm{~K}\end{array}$ & $\begin{array}{l}100.8 \mathrm{~K} \\
51.3 \mathrm{~K}\end{array}$ & $\begin{array}{l}100.2 \mathrm{~K} \\
45.9 \mathrm{~K}\end{array}$ \\
\hline Na3 $(\mathrm{H} \perp \boldsymbol{c})$ & $\begin{array}{l}\mathrm{T}_{\mathrm{C}}^{\text {onset }} \\
\mathrm{T}_{\text {offset }}^{\mathrm{C}}\end{array}$ & $\begin{array}{l}101.4 \mathrm{~K} \\
85.7 \mathrm{~K} \\
\end{array}$ & $\begin{array}{l}101.1 \mathrm{~K} \\
63.3 \mathrm{~K}\end{array}$ & $\begin{array}{l}100.9 \mathrm{~K} \\
53.4 \mathrm{~K}\end{array}$ & $\begin{array}{l}100.4 \mathrm{~K} \\
47.3 \mathrm{~K}\end{array}$ \\
\hline Na4 $(\mathrm{H} \perp \boldsymbol{c})$ & $\begin{array}{l}\mathrm{T}_{\mathrm{C}}^{\text {onset }} \\
\mathrm{T}_{\text {offset }}^{\mathrm{c}}\end{array}$ & $\begin{array}{l}101.0 \mathrm{~K} \\
84.9 \mathrm{~K} \\
\end{array}$ & $\begin{array}{l}100.6 \mathrm{~K} \\
63.5 \mathrm{~K} \\
\end{array}$ & $\begin{array}{l}100.2 \mathrm{~K} \\
54.3 \mathrm{~K} \\
\end{array}$ & $\begin{array}{l}99.9 \mathrm{~K} \\
48.2 \mathrm{~K} \\
\end{array}$ \\
\hline $\mathrm{Na0} \quad(\mathrm{H} \| \boldsymbol{c})$ & $\begin{array}{l}\mathrm{T}_{\mathrm{c}}^{\text {onset }} \\
\mathrm{T}_{\text {offset }}^{\mathrm{c}}\end{array}$ & $\begin{array}{l}100.2 \mathrm{~K} \\
84.2 \mathrm{~K}\end{array}$ & $\begin{array}{l}100.1 \mathrm{~K} \\
72.7 \mathrm{~K}\end{array}$ & $\begin{array}{l}100.0 \mathrm{~K} \\
65.1 \mathrm{~K}\end{array}$ & $\begin{array}{l}99.8 \mathrm{~K} \\
60.4 \mathrm{~K}\end{array}$ \\
\hline $\mathrm{Na} 2 \quad(\mathrm{H} \| c)$ & $\begin{array}{l}\mathrm{T}_{\mathrm{c}}^{\text {onset }} \\
\mathrm{T}_{\text {offset }}^{\mathrm{c}}\end{array}$ & $\begin{array}{l}101.2 \mathrm{~K} \\
85.7 \mathrm{~K} \\
\end{array}$ & $\begin{array}{l}101.6 \mathrm{~K} \\
74.9 \mathrm{~K} \\
\end{array}$ & $\begin{array}{l}101.4 \mathrm{~K} \\
68.1 \mathrm{~K} \\
\end{array}$ & $\begin{array}{l}101.2 \mathrm{~K} \\
64.2 \mathrm{~K} \\
\end{array}$ \\
\hline
\end{tabular}




\section{Figure captions:}

Figure 1. Powder XRD patterns of all samples. Diffraction planes correspond to the Bi-2212 phase. Peaks indicated by *, and + correspond to the $\mathrm{Bi}-2201$, and $\mathrm{SrCuO}_{2}$ phases, respectively

Figure 2. Temperature dependence of resistivity for various applied magnetic fields for $(a)$ pure and $(b) \mathrm{x}=0.10$ samples

Figure 3. Temperature dependence of resistivity of $\mathrm{Na} 2$ sample, for the applied magnetic fields perpendicular and parallel to the $c$-axis

Figure 4. Arrhenius plot of the resistivity of for $(a)$ pure and $(b) \mathrm{x}=0.10$ samples

Figure 5. Flux pinning energy, U, versus applied magnetic field for all the samples

Figure 6. $\Delta \mathrm{T}$ versus magnetic field for all samples

Figure 7. Irreversibility lines of all samples for $\mathrm{T}_{\mathrm{c}}{ }^{\text {offset }}$

Figure 8. Upper critical magnetic field $H_{c 2}(T)$ versus $T c$ for all samples 
Figure 1

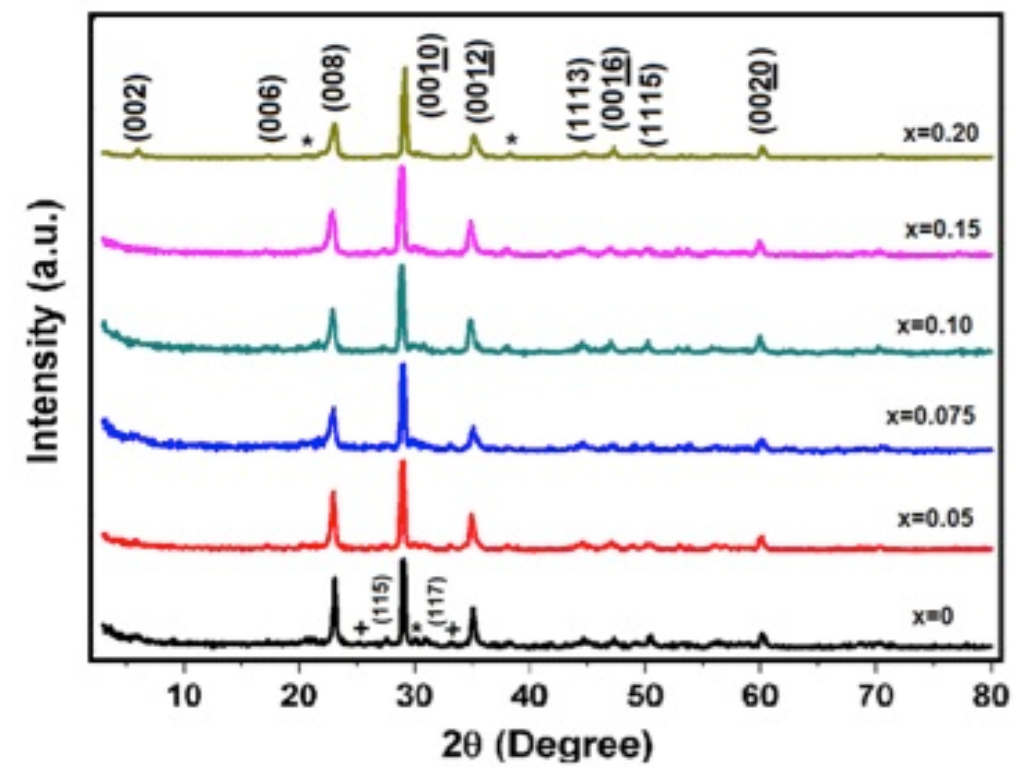


Figure 2
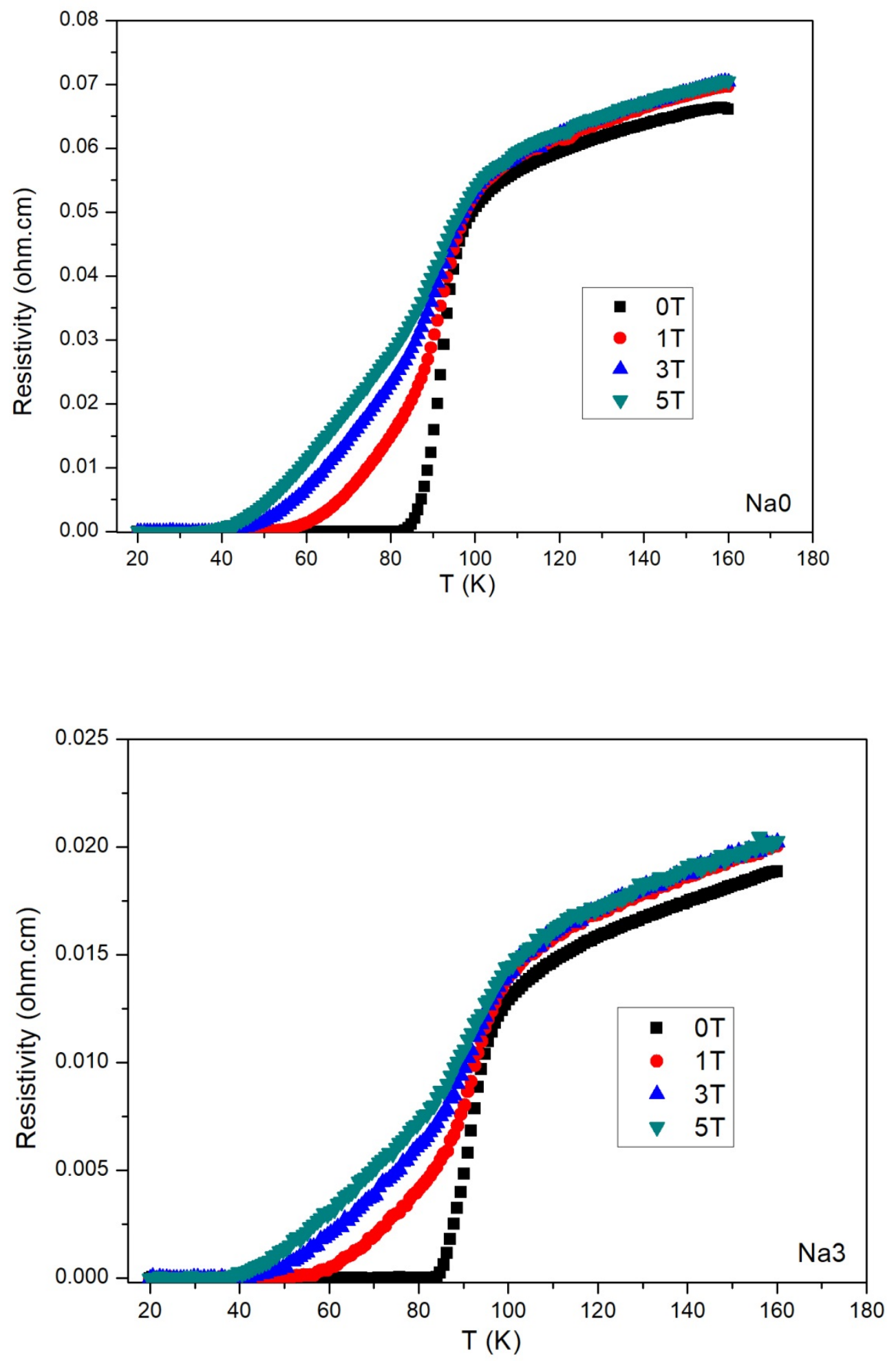
Figure 3

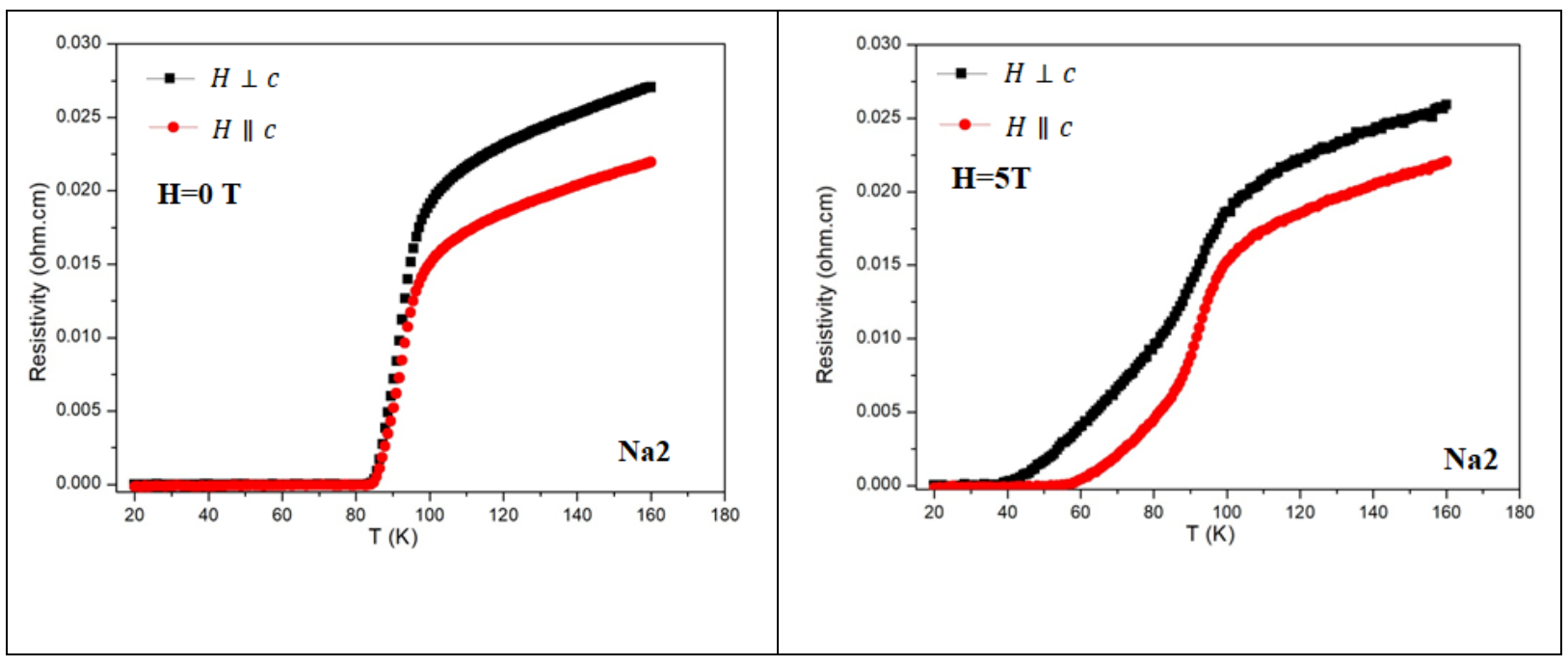


Figure 4
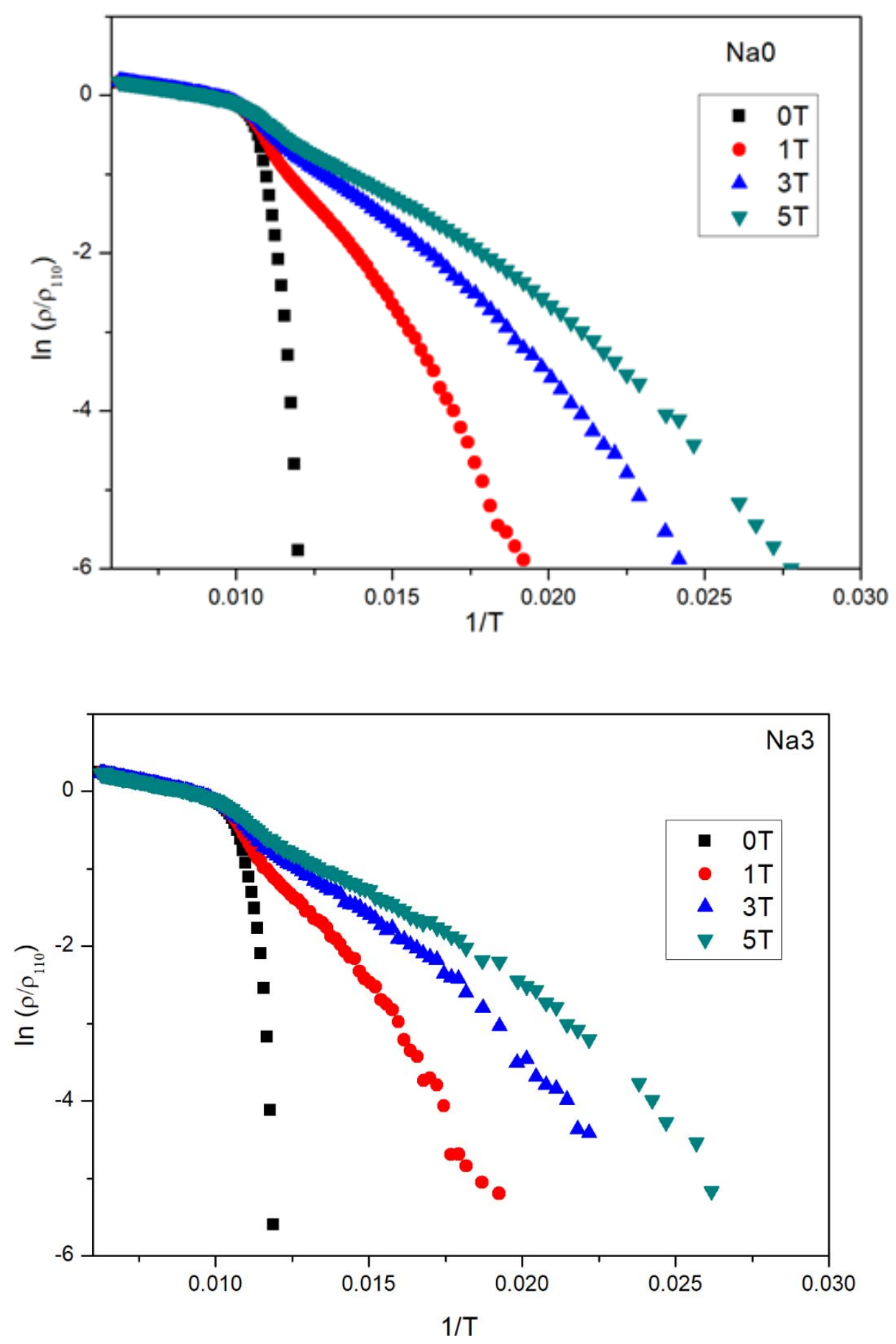
Figure 5

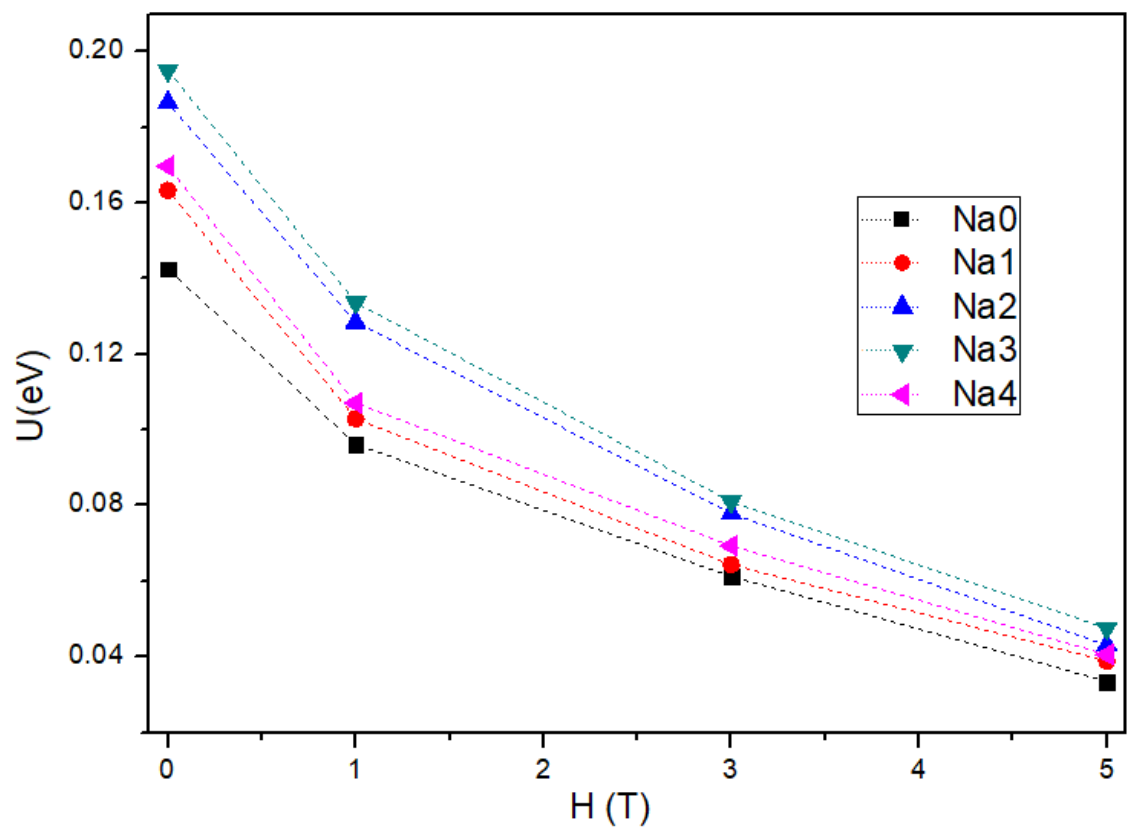


Figure 6

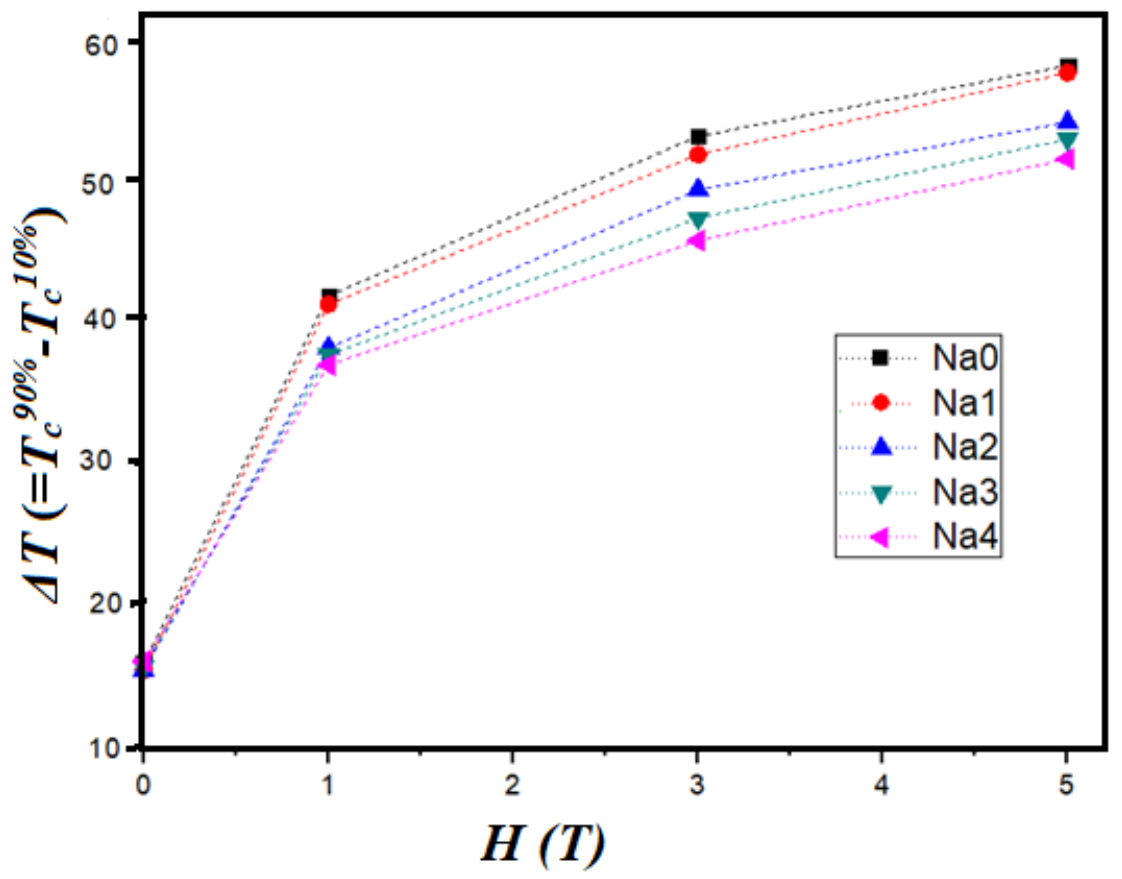


Figure 7

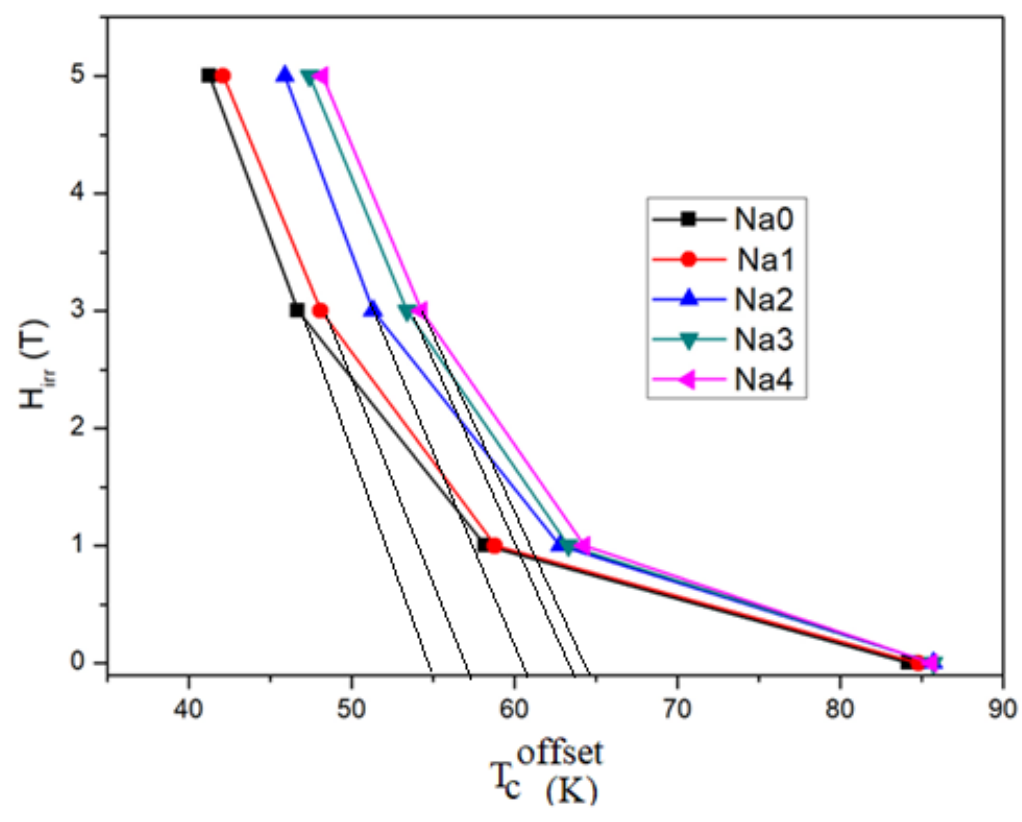


Figure 8

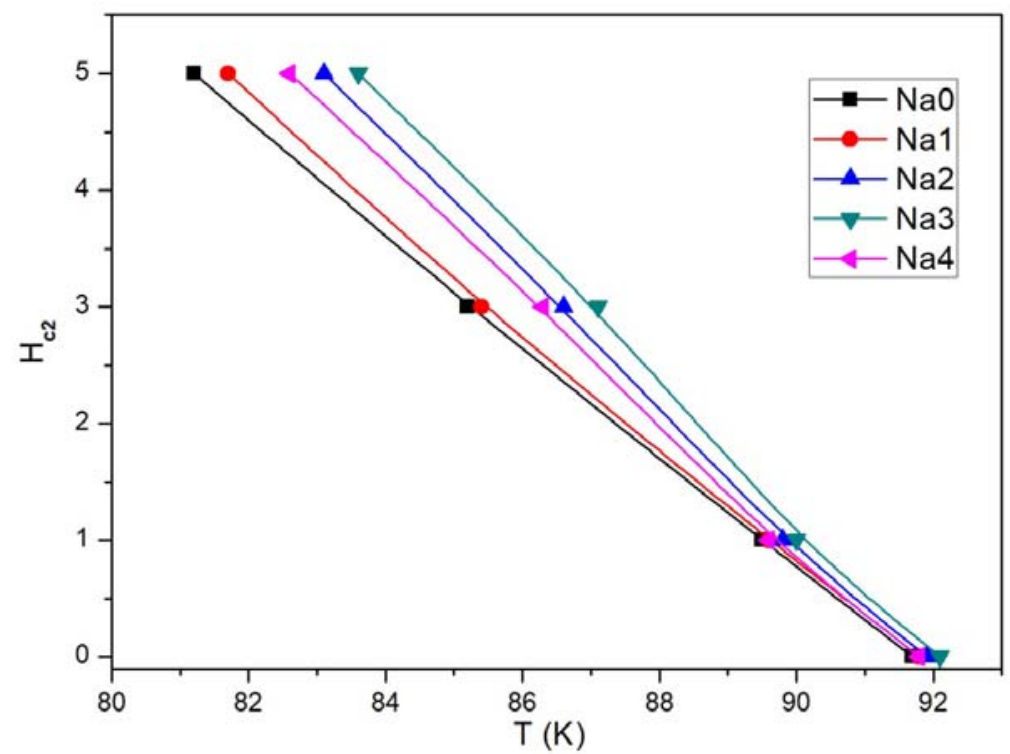

